\title{
Experience on Diagnosis and Treatment of Strangulated Intestinal Obstruction Caused by Mesentery Vein Thrombosis on Account of Portal Hypertension*
}

\author{
Songbing He, Xinguo Zhu\# \\ Department of General Surgery the First Affiliated Hospital of Soochow University, Suzhou, China \\ Email: "captain_hsb@163.com
}

Received 2013

\begin{abstract}
In the present study, to investigate diagnosis and treatment of strangulated intestinal obstruction caused by mesentery vein thrombosis on account of portal hypertension, the data in twelve patients with this disease from 1998 to 2008 were analyzed. All patients presented abdominal pain and vomiting and were confirmed strangulated intestinal obstruction caused by mesentery thrombosis with operation. In this group, nine patients underwent part of small intestine excision, and three patients underwent open-closed operation because of the whole small intestine necrosis caused by intensive mesentery thrombosis. Five patients died after operation. The diagnosis of strangulated intestinal obstruction caused by mesentery thrombosis was difficult because of the slow disease processes and severe outcomes. It is necessary to take some measures to get over the dangers duration after operation.
\end{abstract}

Keywords: Portal Hypertension; Vein Thrombosis; Intestinal Obstruction

\section{Introduction}

The morbidity ratio of strangulated intestinal obstruction caused by vena mesenteric thrombopoiesis pre-operation is much lower than that of post-operation regarding to portal hypertension [1]. In resent 10 years, there are 162 cases of strangulated intestinal obstruction treated in our hospital, among which 12 cases (about 7.4\%) are induced by thrombopoiesis on account of portal hypertension. Here is the analysis of pathogenesis, diagnosis and treatment on the disease.

\section{Clinical Materials}

\section{1) General information}

The clinical sample contains twelve cases in which patients suffer mesentery vein thrombosis caused by portal hypertension induced by posthepatitic cirrhosis. In this group, every patient has a history of type B hepatitis for above five years with the symptoms of splenomegaly and hypersplenism. Five patients have a history of upper digestive tract hemorrhage. Portal hypertension is proved only in two cases by ultrasound, CT scan or/and inter-

\footnotetext{
"Supported by: Science and Technology Research Project of in Science and Technology Bureau of Suzhou City, China (No. SYS201220); Grants from Medical Science and Technology Development Foundation, Jiangsu Province Department of Health, China (No.H201209).

${ }^{\#}$ Corresponding author.
}

ventional radiography (Figure 1), while ascites in the patients were detected in eight cases. In this group, there is only one case in Grade A, eight cases in Grade $\mathrm{B}$ and three cases in Grade C classified by Child-Pugh Grading.

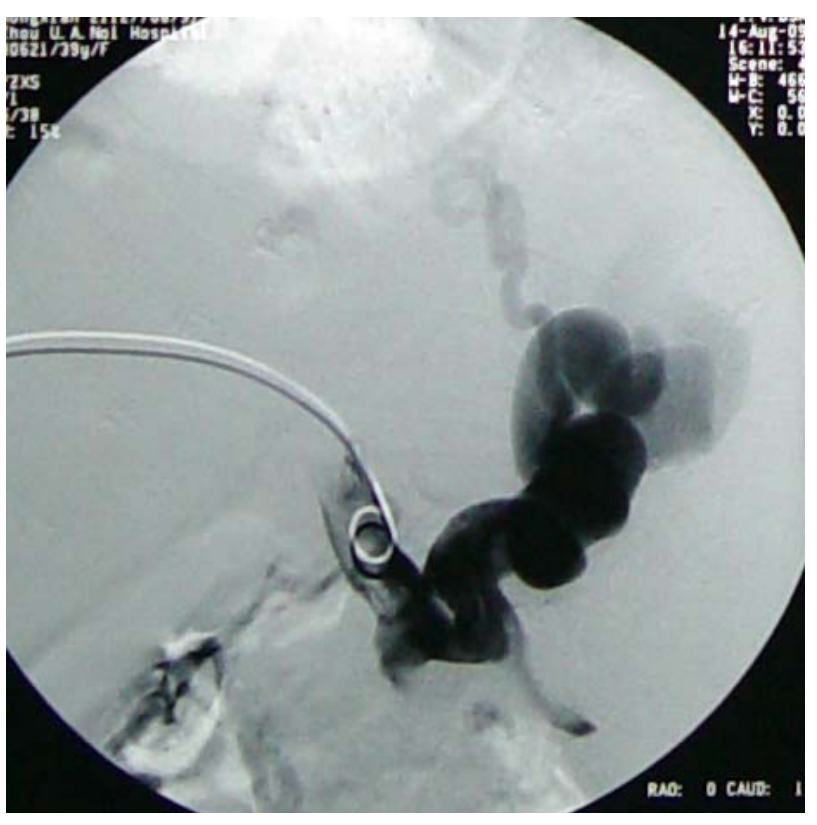

Figure 1. Interventional radiography of mesentery vein thrombosis. 


\section{2) Clinical manifestation}

Among twelve cases, there are six patients presenting irregular or continuous fever of unknown origin between $38.5^{\circ} \mathrm{C}$ to $40.2^{\circ} \mathrm{C}$ lasting for ten to forty-one days. All twelve cases have the symptoms of nausea, vomiting, epigastric gas pains, but only six cases have intense bellyache. The physical sign of peritonitis could be detected in seven patients, and haemic ascites is punctured out from abdominal cavity.

Pneumatics in enteric cavity is displayed in abdominal X-ray plains of all the cases, besides small amounts of gas-fluid level without obviously distended ansa interstinalis can be seen in only three cases.

\section{3) Results}

All cases have to be received emergency exploratory laparotomy. The whole mesostenium which is diffusingly dotted with bleeding points of unequal size are thickened and congested extensively. Among them, two cases are taken to emergency exploratory laparotomy without obvious improvement after conservative treatment in twenty-four hours. Nine cases underwent partial resection of the small intestine due to small intestine, while three cases underwent open-close operation because of full length small intestine and part of colon necrosis simultaneously with a great quantity of haemic ascites. Five cases died after operation in which three cases are widespread thrombosis and two cases are liver function failure after partial resection of the small intestine. Also we can get the immunohistochemistry result of the patients after operation (Figure 2).

\section{Discussion}

The venous thrombosis is concerned with lesion of vas-

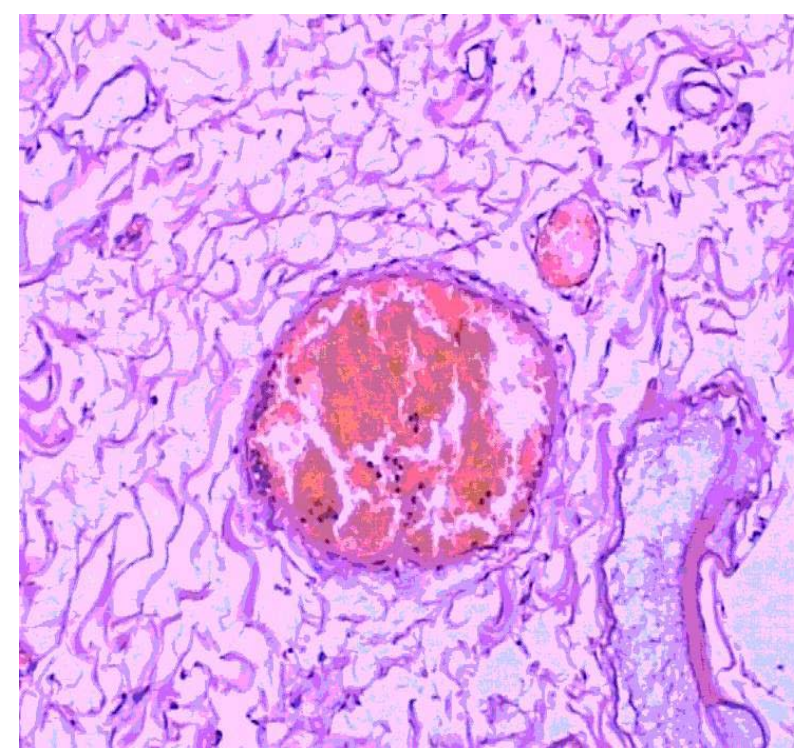

Figure 2. Immunohistochemistry of mesentery vein thrombosis. cular endothelial cell, adhering and assembling of platelets, low serum antithrombase, and alteration of haemodynamics [2,3]. Posthepatitic cirrhosis is a kind of hepatic sinusoid cirrhosis, which has augmented resistance of blood flow returning to liver resulting in hypertension in vena mesenteric and torpidity of blood flow. Meanwhile a bulk of fatty acid, amino acids and carbohydrate absorbed in small intestine are accumulated in mesostenium which increase the viscosity. Chronic portal hypertension plus ischemic and hypoxia of endothelial cells of vena mesenteric could damage endothelial cells unavoidably. The function of single platelet won't be weakened, instead, adhering and assembling of platelets will augment due to the necessity of compensation in spite of the increasing demolition of platelet leading by splenomegaly and hypersplenism. In addition, patients of hepatic cirrhosis with low level of hepatic protein synthesis are in the hypercoagulable state owing to the dysfunction of antiprothrombin [4]. In a word, mesostenium in portal system is prone to thrombopoiesis influenced by all the factors mentioned above.

The disorder of hemorheology been secondary to thrombopoiesis in vena mesenteric which leads to completely interruption of venous blood flow will result in the similar circumstance in the arterial mesenteric, and strangulated intestinal obstruction will emerge ultimately [5,6]. It is different from acute volvulus with the interruption of blood flow both in arterial and vena mesenteric simultaneously causing the typically manifestation such as intense abdominal pain, nausea and vomiting. The symptom of intense abdominal pain occurs only in seven cases in this group, and all the others have non-specificity manifestations such as abdominal discomfort, nausea and vomiting. Furthermore, two cases attempted to adopt conservative treatment before exploratory laparotomy. Thus, the clinical manifestation of strangulated intestinal obstruction caused by vena mesenteric thrombopoiesis on account of portal hypertension is so insidious that it is difficult to have timely and accurate diagnosis. Continuous fever and general toxic symptoms with leucocytes count of more than $20 \times 10^{9}$ per liter would emerge attaching to vena mesenteric thrombopoiesis. What's more, ascites even jaundice and alimentary tract hemorrhage could be detected if infective pylephlebitis occurs in addition to the aggravation of infective symptoms. Accordingly, it is necessary to take ultrasound or CT scan for patients of portal hypertension with the symptoms of continuous fever, abdominal pain, abdominal distention, nausea and vomiting. Thrombopoiesis in vena mesenteric, thickened mesostenium, and haemic ascites can be considered as sufficient indications of emergency exploratory laparotomy [7]. Actually there are three cases in this group dying from extensive thrombopoiesis owing to delayed diagnosis. 
Since the residual embolic mesostenium is the original cause of fever and thrombopoiesis after emergency exploratory laparotomy, pathologic mesostenium has to be resected thoroughly when removing the necrosis intestine for patients of the strangulated intestinal obstruction caused by vena mesenteric thrombopoiesis on account of portal hypertension. Meanwhile, adjunctive therapy such as protection of hepatic function, nutritional support, diureses, and proper blood transfusion calls for more attention as most patients in emergency operations occupying a hepatic inadequacy state of Grade B even C.

\section{Acknowledgements}

We would like to express our thanks to the anonymous reviewers for their suggestions, which helped to improve this paper.

\section{REFERENCES}

[1] M. C. Wang, S. Li, J. Y. Zhu, X. S. Leng and R. Y. Du, "The Reason and Treatment of Portal Vein Thrombosis in Patients with Portal Hypertension Postoperation,” Zhonghua Wai Ke Za Zhi, Vol. 42, 2004, pp. 269-271.

[2] J. Turnes, J. C. García-Pagán, M. González, C. Aracil, J. L. Calleja, C. Ripoll, J. G. Abraldes, R. Bañares, C. Villanueva, A. Albillos, J. R. Ayuso, R. Gilabert and J. Bosch, "Portal Hypertension-Related Complications after Acute Portal Vein Thrombosis: Impact of Early Anticoa- gulation,” Clinical Gastroenterology and Hepatology, Vol. 6, 2008, pp. 1412-1417.

http://dx.doi.org/10.1016/j.cgh.2008.07.031

[3] O. Harmanci and Y. Bayraktar, "Portal Hypertension Due to Portal Venous Thrombosis: Etiology, Clinical Outcomes," World Journal of Gastroenterology, Vol. 13, 2007, pp. 2535-2540.

[4] C. Vivian, McAlister, A. Noha and Al-Saleh, "Duodenal Dearterialization and Stapling for Severe Hemorrhage from Duodenal Varices with Portal Vein Thrombosis," The American Journal of Surgery, Vol. 189, 2005, pp. 4952. http://dx.doi.org/10.1016/j.amjsurg.2004.04.011

[5] O. Ateş, G. Hakgüder, M. Olguner, M. Seçil, I. Karaca and F. M. Akgür, "Mesenterico Left Portal Bypass for Variceal Bleeding Owing to Extrahepatic Portal Hypertension Caused by Portal Vein Thrombosis," Journal of Pediatric Surgery, Vol. 41, 2006, pp. 1259-1263. http://dx.doi.org/10.1016/j.jpedsurg.2006.03.043

[6] A. C. Chin, F. Thow and R. A. Superina, "Previous Portal Hypertension Surgery Negatively Affects Results of Mesenteric to Left Portal Vein Bypass," Journal of Pediatric Surgery, Vol. 43, 2008, pp. 114-119. http://dx.doi.org/10.1016/j.jpedsurg.2007.09.032

[7] Y. M. Galeev, Y. B. Lishmanov, E. G. Grigorev, M. V. Popov, K. A. Aparcin and O. V. Salato, "Scintigraphic Visualization of Bacterial Translocation in Experimental Strangulated Intestinal Obstruction," European Journal of Nuclear Medicine and Molecular Imaging, Vol. 36, 2009, pp. 1822-1828.

http://dx.doi.org/10.1007/s00259-009-1146-5 